\title{
Hermanlentia n. gen. da tribo Triatomini, com um rol de espécies de Triatominae (Hemiptera, Reduviidae)
}

\author{
José Jurberg ${ }^{+}$, Cleber Galvão
}

\begin{abstract}
Laboratório Nacional e Internacional de Referência em Taxonomia de Triatomíneos, Departamento de Entomologia, Instituto Oswaldo Cruz, Av. Brasil 4365, 21045-900 Rio de Janeiro, RJ, Brasil
\end{abstract}

Hermanlentia n. gen. of Tribe Triatomini, with a List of Species of Triatominae (Hemiptera, Reduviidae) - Hermanlentia $n$. gen. was established in the tribe Triatomini, based on differences of external morphology, phallic structures and chromatic characters of Triatoma matsunoi FernandezLoayza 1989, in relation to other species of Triatoma. The head is long with disproportionally small eyes 1:0,16; the relation of the head and the pronotum is 1:0,4; and the coloration of the hemelytron is whitish. Characters of the male genitalia that distinguish the new genus are the articulatory apparatus with twice the size of the aedeagus, phallosoma with fingerlike apex, $1+1$ dorsal conjuntive processes with the inner edge toothed, and absence of endosoma process and vesica.

Key words: Hermanlentia n. gen. - Triatoma matsunoi - Triatominae

Em 1993 Lent et al. descreveram detalhes morfológicos mais amplos de Triatoma matsunoi Fernandez-Loayza, 1989, principalmente os relacionados às estruturas da genitália externa masculina, do que aqueles publicados por seu autor e a consideraram como "uma das espécies atípicas do gênero".

Baseados na observação feita, foram assinalados detalhes morfológicos, cromáticos e fálicos até o presente não encontrados em outras espécies de Triatoma, isto é, hemélitros, principalmente as membranas, nos dois sexos, sem o colorido habitual, são esbranquiçadas e as nervuras sem contraste, a nervura $\mathrm{M}$ da asa anterior é incompleta, e as patas são alongadas. Comparandoa com a espécie tipo do gênero, Triatoma rubrofasciata (De Geer, 1773), veremos que além das diferenças externas (Lent \& Wygodzinsky 1979), encontramos mais seis diferenças marcantes (veja quadro comparativo das estruturas fálicas dos gêneros de Triatomini) em relação à genitália masculina (Lent \& Jurberg 1978, Lent et al. 1993a, b): (1) aparelho articular bastante desenvolvido em T. matsunoi, duas vezes mais longo que o edeago; em $T$. rubrofasciata a proporção é de $1: 1$; (2)

Com auxílio do CNPq, convênio BIRD/FNS/FIOCRUZ 027/93 e Commission of the European Communities TS3 CT 920092.

+Autor de contato. Fax: +55-21-290.9339. E-mail: galvao@dcc001.cict.fiocruz.br.

Recebido em 3 de abril de 1996

Aceito em 7 de janeiro de 1997 processos da conjuntiva: presença de $1+1$, localizados dorsalmente, com bordos internos denteados, situados no ápice do edeago em $T$. matsunoi e ausentes em T. rubrofasciata; (3) processos do endosoma ausentes em T. matsunoi, presentes em $T$. rubrofasciata; (4) vesica, ausente em T. matsunoi e presente em T. rubrofasciata; (5) falosoma com ápice digitiforme em T. matsunoi, e arredondado em T. rubrofasciata; (6) processo mediano do pigóforo com ápice escamoso em $T$. matsunoi e liso em $T$. rubrofasciata (ver quadro comparativo).

\section{Hermanlentia $\mathrm{n}$. gen.}

Gênero monotípico - Espécie tipo: Triatoma matsunoi Fernandez-Loayza, 1989. Morfometria: baseada em 1 macho e 1 fêmea (tipos). Comprimento total do macho: $29 \mathrm{~mm}$, da fêmea: $31 \mathrm{~mm}$. Cabeça muito longa e olhos pouco salientes, desproporcionais em relação ao comprimento da cabeça 1:0,16; comprimento da cabeça: $6 \mathrm{~mm}$, região ante-ocular: $4,1 \mathrm{~mm}$, região pós-ocular: 0,9 $\mathrm{mm}$, região interocular: $1,1 \mathrm{~mm}$, largura da cabeça ao nível dos olhos: $2 \mathrm{~mm}$, largura do olho em vista dorsal: $0,4 \mathrm{~mm}$, comprimento do pronoto: $4 \mathrm{~mm}$, largura do pronoto: 5,2 mm. Relação entre cabeça e pronoto: 1:0,6 (Figs. 1, 2, 3).

O falo é constituído por um aparelho articular com o dobro do tamanho do edeago; processos da conjuntiva situados dorsalmente e no ápice do edeago com bordos internos denticulados; vesica e processos do endosoma ausentes; falosoma com ápice digitiforme e processo mediano do pigóforo com superfície escamosa (Figs. 3 a 9 de Lent et al. 1993b). 
Quadro comparativo das estruturas fálicas dos gêneros de Triatomini

\begin{tabular}{|c|c|c|c|c|c|c|c|c|c|c|}
\hline & PB & PrG & EPlb & $\mathrm{Ph}$ & $\mathrm{PrPh}$ & $\mathrm{SPh}$ & $\mathrm{PrCj}$ & PrEn & $\mathrm{V}$ & PrP \\
\hline Triatoma & + & + & + & + & - & + & - & $\begin{array}{c}1+1 \\
+\end{array}$ & + & + \\
\hline Hermanlentia & + & $\begin{array}{c}\text { muito longc } \\
+\end{array}$ & $\begin{array}{c}\text { muito longo } \\
+\end{array}$ & $\begin{array}{c}\text { ápice } \\
\text { digitiforme } \\
+\end{array}$ & - & + & $\begin{array}{c}1+1 \\
\text { com dentes } \\
+\end{array}$ & - & - & + \\
\hline Mepraia & + & + & + & + & - & - & $\begin{array}{c}1+1 \\
\text { aliforme } \\
+ \\
\end{array}$ & - & $\begin{array}{c}\operatorname{arco} \\
+\end{array}$ & + \\
\hline Eratyrus & + & + & + & + & - & + & - & + & + & + \\
\hline Dipetalogaster & + & + & + & + & - & + & - & $\begin{array}{c}\text { impar } \\
\text { pequeno } \\
+\end{array}$ & + & + \\
\hline Panstrongylus & + & + & + & + & - & + & - & $\begin{array}{c}1+1 \\
+\end{array}$ & + & + \\
\hline Paratriatoma & + & + & + & + & - & + & - & $\begin{array}{c}1+1 \\
+\end{array}$ & + & + \\
\hline Linshcosteus & + & + & + & + & - & + & - & $\begin{array}{c}1+1 \\
+\end{array}$ & + & + \\
\hline
\end{tabular}

Eplb: extensão mediana da placa basal; PB: ponte basal; Ph: falosoma; PrCj: processo da conjuntiva; PrEn: processo do endosoma; PrG: processo do gonoporo; PrP: processo do pigóforo; $\mathrm{PrPh}$ : processo do falosoma; $\mathrm{SPh}$ : suporte do falosoma; V: vesica.

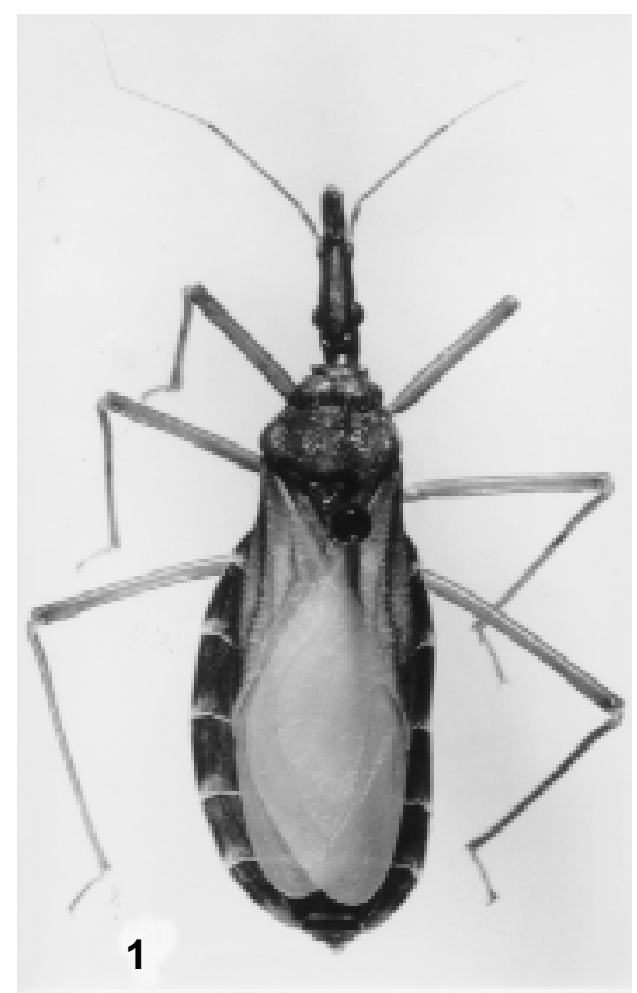

Fig. 1: Hermanlentia matsunoi (Fernandez-Loayza, 1989).
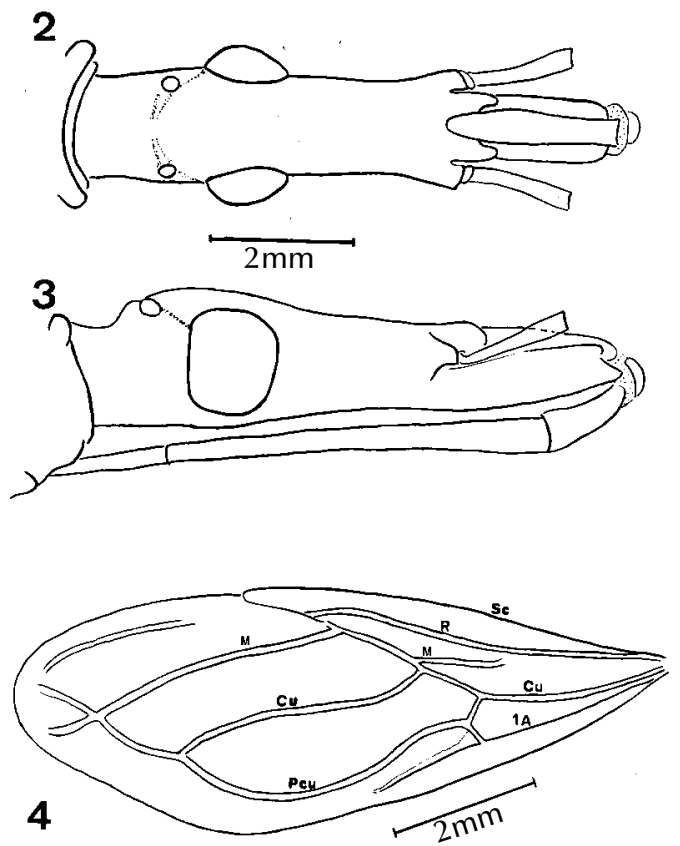

Hermanlentia matsunoi (Fernandez-Loayza, 1989) - Figs. 2,3: cabeça vista dorsal e ventral. Fig. 4: asa anterior. Abreviaturas: Sc: subcostal, R: radial, M: mediana, $\mathrm{Cu}$ : cubital, Pcu: poscubital, 1A: primeira anal. 
A forma e a presença e/ou ausência das estruturas comparadas com os outros gêneros de Triatomini mostra o seguinte: (1) a proporção entre o aparelho articular com o dobro do tamanho do edeago é única para Hermanlentia em relação aos demais gêneros; (2) a presença de processos na conjuntiva só ocorre em Mepraia Mazza, Gajardo \& Jorg,1940 localizados lateralmente e estriados; em Hermanlentia são dorsais com bordos internos denteados; (3) a ausência de processos do endosoma também só ocorre em Mepraia e em Dipetalogaster Usinger, 1939 onde é ímpar e pequeno; (4) a ausência da vesica somente ocorre em Hermanlentia (Quadro).

Distribuição geográfica: Peru, Departamento de La Libertad, Província Pataz, Distrito Piaz.

Tipos: holótipo no 2831 e alotipo no 2832 depositados na Coleção Entomológica do Instituto Oswaldo Cruz - Coleção Herman Lent de Triatomíneos que alberga atualmente 37 holótipos, 15 alótipos e 192 parátipos no seu acervo (Gonçalves et al. 1993).

O nome genérico é dedicado a Herman Lent, zoólogo brasileiro, nascido em 3 de fevereiro de 1911 na cidade do Rio de Janeiro, pesquisador do Instituto Oswaldo Cruz e da Universidade Santa Úrsula, membro titular da Academia Brasileira de Ciências, que desde 1935 se dedica ao estudo dos triatomíneos, vetores da doença de Chagas (Jurberg 1993a, b), após consulta ao Nomenclator Zoologicus (Neave 1939, 1950).

\section{DISCUSSÃO}

Através da forma das estruturas fálicas, associadas à morfologia externa, tem sido possível caracterizar cada espécie, bem como situá-las entre as espécies afins (Jurberg 1991). Fundamentados na presença ou ausência e tendo como base dez estruturas fálicas aliados à forma das estruturas, foi possível separar os gêneros, tribos e conjeturar-se sobre a elaboração da filogenia desta subfamília. Esta abordagem deverá associar os conhecimentos morfológicos a outros parâmetros (Jurberg 1978, 1995).

Davis, em 1961, analisando o modelo das nervuras nas asas anteriores e posteriores fez um estudo abrangente entre os Reduvioidea visando a criação de um parâmetro a ser incorporado ao estudo filogenético; porém entre os triatomíneos só Triatoma protracta (Uhler) teve as nervuras das asas anteriores e posteriores nominadas. Desde 1967 quando Lent e Jurberg analisaram as nervuras da asa anterior de Mepraia spinolai (Porter, 1934) (Lent, Jurberg \& Galvão, 1994), outras três espécies de cinco gêneros foram analisadas: Panstrongylus geniculatus (Latreille, 1811), Eratyrus mucronatus Stal, 1859 e Dipetalogaster maxima Usinger, 1939.
Confrontando-se com H. matsunoi nota-se que só nesta última a nervura $\mathrm{M}$ da asa anterior não é completa (Lent \& Jurberg, 1968, 1970, 1972).

Em relação aos caractéres da genitália masculina comparamos as estruturas fálicas desta espécie com as demais do gênero Triatoma (Lent \& Jurberg 1967, 1978, 1980, 1981, 1982, 1985, 1987, 1992, Lent et al. 1993, 1994b e 1995) e com as dos outros gêneros de Triatomini (Lent \& Jurberg 1968, 1970, 1971, 1972, 1975, 1983, Lent et al. 1994a) fundamentados nas diferenças externas já assinaladas e nas encontradas na genitália.

Atualmente a subfamília Triatominae está constituída de 123 espécies pois, desde 1979, quando Lent e Wygodzinsky publicaram uma revisão do grupo foram adicionadas nove espécies novas, um gênero e uma espécie foram revalidados e uma subespécie elevada a categoria de espécie.

1 - Alberprosenia malheiroi Serra, Atzingen \& Serra, 1987

Tipos: Fac. Saúde Pública USP, Coleção Carcavallo no Instituto Oswaldo Cruz, 2 parátipos Distribuição: Pará, Brasil

Observações: esta espécie foi apresentada no V Congresso Brasileiro de Parasitologia em 1980. Carcavallo (1987) publicou uma tradução do resumo do congresso divulgando formalmente a espécie para a comunidade científica, com uma foto.

2 - Belminus laportei Lent, Jurberg \& Carcavallo, 1995

Tipos: holótipo - macho, Utinga, Belém, $\mathrm{n}^{0}$ 2133; alótipo - fêmea, Monte Dourado, Pará, no 2132; ambos depositados na Coleção Herman Lent do Instituto Oswaldo Cruz, Rio de Janeiro, Brasil Distribuição: Belém, Pará, Brasil.

3 - Belminus pittieri Osuna \& Ayala,1993 sp. inq. Tipos: holótipo - fêmea, Rancho Grande, Aragua, depositado na Colección de Insectos, Francisco Fernandéz Yépez del Museo del Instituto de Zoologia Agricola (MIZA)

Distribuição: Rancho Grande, Edo. Aragua, Venezuela

Observações: coletado a $1050 \mathrm{~m}$ de altitude.

4 - Cavernicola lenti Barrett \& Arias, 1985 Tipos: holótipo - macho, Balbina, Amazonas, $\mathrm{n}^{\mathrm{o}}$ 1992; alótipo - fêmea, Balbina, Amazonas, no 1993; ambos depositados na Coleção Herman Lent do Instituto Oswaldo Cruz, Rio de Janeiro, Brasil Distribuição: Amazonas, Brasil (Hidrelétrica de Balbina, Rio Uatumã)

Biologia: encontrado em ocos de árvores associados a Eratyrus mucronatus Stal (Triatominae), Rhipidomys sp. (Rodentia) e Phyllostomus 
elongatus Geoffroy (Micro-chiroptera), infectado com Trypanosoma cruzi.

5 - Mepraia spinolai Mazza, Gajardo \& Jorg, 1940: Lent, Jurberg \& Galvão, 1994a

Tipos: holótipo: Instituto Oswaldo Cruz, Rio de Janeiro, Brasil

Distribuição: Chile, em regiões semi-áridas e áridas desde $3000 \mathrm{~m}$ de altura até o nível do mar

Biologia: suga aves, mamíferos e répteis a qualquer hora do dia, inclusive sob a luz solar; vive entre as pedras ou nos locais onde os animais se abrigam; encontrado naturalmente infectado pelo $T$. cruzi.

6 - Rhodnius stali Lent, Jurberg \& Galvão, 1993a Tipos: holótipo macho, Salobra, Mato Grosso, Brasil, no 645; alótipo fêmea, Bodoquena, Mato Grosso, Brasil, no 647; ambos depositados na Coleção Herman Lent do Instituto Oswaldo Cruz, Rio de Janeiro, Brasil

Distribuição: Mato Grosso, Brasil, Bolívia.

7 - Triatoma bolivari Carcavallo, Martinez \& Pelaez, 1987

Tipos: holótipo macho, Colecão Rodolfo Carcavallo no Instituto Oswaldo Cruz , 5 parátipos Distribuição: Colima, Nayarit, Jalisco, México Biologia: espécie peridomiciliar e silvestre sempre capturada atraída pela luz; encontrada naturalmente infectada por flagelados semelhantes ao T. cruzi.

8 - Triatoma brailovskyi Martínez, Carcavallo \& Pelaez,1984

Tipos: holótipo macho, Colecão Rodolfo Carcavallo no Instituto Oswaldo Cruz

Distribuição: Colima, Jalisco e Nayarit, México.

9 - Triatoma bruneri (Usinger,1944): Lent \& Jurberg, 1981

Tipos: California Academy of Sciences Distribuição: Cuba

Observações: até há pouco esta espécie era considerada sinônimo de T. flavida (Usinger, 1944).

10 - Triatoma gomeznunezi Martinez, Carcavallo \& Jurberg, 1994

Tipos: holótipo - macho, Candelaria Loxica, Portillo del Rayo, Oaxaca, México depositado na Coleção Rodolfo Carcavallo no Instituto Oswaldo Cruz

Distribuição: Oaxaca, México

Observações: o único espécime conhecido foi atraído pela luz e capturado em uma floresta tropical.

\section{1 - Hermanlentia matsunoi (Fernandez-Loayza,} 1989)

Tipos: holótipo - fêmea, Corralon, Pias, Pataz, Peru, no 2831; alótipo - macho, Corralon, Pias, Pataz, Peru, no 2832; ambos depositados na Coleção
Herman Lent do Instituto Oswaldo Cruz, Rio de Janeiro, Brasil

Distribuição: Pataz, La Libertad, Peru

Biologia: os espécimes coletados pelo autor da espécie não estavam infectados por flagelados semelhantes ao T. cruzi.

12 - Triatoma melanosoma (Martinez, Olmedo \& Carcavallo, 1987) Lent, Jurberg, Galvão \& Carcavallo, 1993

Tipos: Coleção Rodolfo Carcavallo no Instituto Oswaldo Cruz

Distribuição: Misiones, Argentina

Observações: descrita originalmente como subespécie, Triatoma infestans melanosoma.

\section{REFERÊNCIAS}

Barrett TV, Arias JR 1985. A new Triatominae host of Trypanosoma from the Central Amazon of Brazil: Cavernicola lenti n. sp.(Hemiptera, Reduviidae, Triatominae). Mem Inst Oswaldo Cruz 80: 91-96.

Carcavallo RU 1987. Nueva especie del género Alberprosenia Martinez y Carcavallo, 1977 del Estado de Pará, Brasil (Hemiptera, Triatominae) Comentário editorial. Chagas 4: 3.

Carcavallo RU, Rabinovich J, Tonn RJ 1985. Factores biologicos y ecologicos em la enfermedad de Chagas. Tomo II Chagas, número especial, 472 pp.

Carcavallo R, Martínez A, Peláez D 1987. Una nueva especie de Triatoma Laporte de México. Chagas 4: 476-477.

Davis NT 1961. Morphology and Phylogeny of the Reduvidoidea (Hemiptera:Heteroptera). Part II Wing venation. Ann Entomol Soc America 54: 340-354.

Fernandez-Loayza R 1989. Triatoma matsunoi nueva especie del norte peruano (Hemiptera, Reduviidae: Triatominae). Rev per Ent 31: 21-24.

Gonçalves TCM, Almeida MD, Jurberg J, Lent H 1993. Lista dos exemplares-tipos de triatomíneos depositados na Coleção Entomológica do Instituto Oswaldo Cruz, Rio de Janeiro (Hemiptera: Reduviidae). Mem Inst Oswaldo Cruz 88: 327-333.

Jurberg J 1978. Contribuição ao estudo comparativo da genitália externa em subfamílias de Reduviidae (Hemiptera-Heteroptera). Tese de Mestrado. Univ Federal do Rio de Janeiro, 72 pp.

Jurberg J 1991. A taxonomia dos triatomíneos baseada nas estruturas fálicas. X Congresso Latino Americano de Parasitologia - I Congresso Uruguayo de Parasitologia. Montevideo, p. 217.

Jurberg J 1993a. Herman Lent - Biografia. Rev bras Zool 10: 1-4.

Jurberg J 1993b. Lista de publicações de Herman Lent. Rev bras Zool 10: 5-17.

Jurberg J 1995. Uma abordagem filogenética entre os triatomíneos baseada nas estruturas fálicas (Hemiptera, Reduviidae). Mem Inst Oswaldo Cruz 90 (Suppl. 1): 59.

Lent H, Jurberg J 1967. Algumas informações sôbre Triatoma spinolai Porter, 1934 com um estudo sôbre as genitálias externas (Hemiptera, Reduviidae). Rev 
Brasil Biol 27: 273-288.

Lent H, Jurberg J 1968. Estudo morfológico comparativo de Panstrongylus geniculatus Latreille, $1911 \mathrm{e}$ Panstrongylus megistus (Burmeister,1835) e suas genitálias externas (Hemiptera, Reduviidae, Triatominae). Rev Brasil Biol 28: 499-520.

Lent H, Jurberg J 1970. O gênero Eratyrus Stal, 1859 com um estudo sobre a genitália externa (Hemiptera, Reduviidae, Triatominae). Rev Brasil Biol 30: 297312.

Lent H, Jurberg J 1971. O gênero Paratriatoma Barber, 1938 com um estudo sobre a genitália externa (Hemiptera, Reduviidae, Triatominae). Rev Brasil Biol 31: 39-41.

Lent H, Jurberg J 1972. O gênero Dipetalogaster Usinger, 1939 com um estudo sobre a genitália externa (Hemiptera, Reduviidae,Triatominae). Studia Entomologica 15: 465- 484.

Lent H, Jurberg J 1975. O gênero Panstrongylus Berg, 1879 com um estudo sobre a genitália externa das espécies (Hemiptera, Reduviidae,Triatominae). Rev Brasil Biol 35: 379-438.

Lent H, Jurberg J 1978. Estudo comparativo da genitália externa masculina de seis espécies de Triatoma Laporte, 1832 que mais frequientemente habitam o domicílio humano no Brasil (Hemiptera, Reduviidae). Rev Brasil Biol 38: 931-944.

Lent H, Jurberg J 1980. Comentários sobre a genitália externa masculina em Triatoma Laporte, 1832 (Hemiptera, Reduviidae). Rev Brasil Biol 40: 611627.

Lent H, Jurberg J 1981. As espécies insulares de Cuba do gênero Triatoma (Hemiptera, Reduviidae). Rev Brasil Biol 41: 431-439.

Lent H, Jurberg J 1982. A genitália externa dos machos no complexo Triatoma phyllosoma (Burmeister). Espécies mexicanas transmissoras da doença de Chagas. Mem Inst Oswaldo Cruz 77: 299-317.

Lent H, Jurberg J 1983. Estudo comparativo da genitália externa masculina de Linshcosteus costalis Ghauri e L. kali Lent \& Wygodzinsky,1979 (Hemiptera, Reduviidae, Triatominae). Mem Inst Oswaldo Cruz, 78: 421-429.

Lent H, Jurberg J 1985. Sobre a variação intra-específica em $T$. dimidiata (Latreille) e T. infestans (Klug) (Hemiptera, Reduviidae). Mem Inst Oswaldo Cruz, 80: 285-299.

Lent H, Jurberg J 1987. A genitália externa dos machos de sete espécies de Triatoma Laporte, 1832 da região Neártica (Hemiptera, Reduviidade). Mem Inst Oswaldo Cruz 88: 227-246.

Lent H, Jurberg J 1992. Estudo comparativo da genitália externa masculina de Triatoma neotomae Neiva,1911 e Triatoma nitida Usinger,1939 (Hemiptera, Reduviidae). Mem Inst Oswaldo Cruz 87: 123-130.

Lent H, Wygodzinsky P 1979. Revision of Triatominae
(Hemiptera, Reduviidae), and their significance as vectors of Chagas'disease. Bull Am Mus Nat Hist 163: $123-520$.

Lent H, Jurberg J, Carcavallo RU 1995. Belminus laportei sp. n. da região Amazônica (Hemiptera: Reduviidae: Triatominae). Mem Inst Oswaldo Cruz 90: 33-39.

Lent H, Jurberg J, Galvão C 1993a. Rhodnius stali n. sp. afim de Rhodnius pictipes Stal,1872 (Hemiptera, Reduviidae, Triatominae). Mem Inst Oswaldo Cruz, 88: 605- 614.

Lent H, Jurberg J, Galvão C 1993b. Triatoma matsunoi Fernandez-Loayza,1989 com um estudo sobre a genitália externa masculina (Hemiptera, Reduviidae,Triatominae). MemInst Oswaldo Cruz 88: 615619.

Lent H, Jurberg J, Galvão C 1994a. Revalidação do gênero Mepraia Mazza, Gajardo \& Jorg, 1940 (Hemiptera, Reduviidae, Triatominae). Mem Inst Oswaldo Cruz 89: 347-352.

Lent H, Jurberg J, Galvão C 1995. Um sinônimo de Triatoma melanosoma Martinez, Olmedo \& Carcavallo 1987 (Hemiptera, Reduviidae). Entomologia y Vectores 2: 81-82.

Lent H, Jurberg J, Galvão C, Carcavallo RU 1994b. Triatoma melanosoma novo status para Triatoma infestans melanosoma Martinez, Olmedo \& Carcavallo,1987 (Hemiptera, Reduviidae). Mem Inst Oswaldo Cruz 89: 353-358.

Martinez A, Carcavallo RU, Jurberg J 1994. Triatoma gomeznunezi a new species of Triatomini from Mexico (Hemiptera, Reduviidae,Triatominae). Entomologia y Vectores 1: 15-19.

Martinez A, Carcavallo RU, Pelaez D 1984. Triatoma brailovskyi nueva espécie de Triatominae de México. Chagas 1: 39-42.

Martinez A, Olmedo RA, Carcavallo RU 1987. Una nueva subespecie Argentina de Triatoma infestans. Chagas 4: 479-480.

Neave SA 1939. Nomenclator Zoologicus. A list of the names of genera and sub-genera in Zoology from tenth Edition of Linnaeus 1758 to the end 1935. 4 vols. Sheffield Airey Neave, The Zoological Society of London.

Neave SA 1950. Nomenclator Zoologicus. A list of the names of genera and sub-genera in Zoology vol. 5, 1936-1945, The Zoological Society of London, 308 pp.

Osuna E, Ayala, JM 1993. Belminus pittieri nueva especie de Bolboderini (Triatominae: Reduviidae: Heteroptera). Bol Entomol Vez N S 8: 147-150.

Serra RG, Atzingen NCB, Serra OP 1987. Nueva especie del género Alberprosenia Martínez y Carcavallo, 1977, del Estado de Pará, Brasil (Hemiptera, Triatominae). Chagas 4: 475. (Traduccíon de un trabajo de 1980). 
